\title{
COMMUTING SEMIGROUPS OF HOLOMORPHIC MAPPINGS
}

\author{
M. ELIN, M. LEVENSHTEIN, S. REICH and D. SHOIKHET
}

\begin{abstract}
Let $S_{1}=\left\{F_{t}\right\}_{t \geq 0}$ and $S_{2}=\left\{G_{t}\right\}_{t \geq 0}$ be two continuous semigroups of holomorphic self-mappings of the unit disk $\Delta=\{z:|z|<1\}$ generated by $f$ and $g$, respectively. We present conditions on the behavior of $f$ (or $g$ ) in a neighborhood of a fixed point of $S_{1}\left(\right.$ or $S_{2}$ ), under which the commutativity of two elements, say, $F_{1}$ and $G_{1}$ of the semigroups implies that the semigroups commute, i.e., $F_{t} \circ G_{s}=G_{s} \circ F_{t}$ for all $s, t \geq 0$. As an auxiliary result, we show that the existence of the (angular or unrestricted) $n$-th derivative of the generator $f$ of a semigroup $\left\{F_{t}\right\}_{t \geq 0}$ at a boundary null point of $f$ implies that the corresponding derivatives of $F_{t}, t \geq 0$, also exist, and we obtain formulae connecting them for $n=2,3$.
\end{abstract}

\section{Introduction}

We denote by $\operatorname{Hol}(\Delta, D)$ the set of all holomorphic functions on the unit disk $\Delta=\{z:|z|<1\}$ which map $\Delta$ into a domain $D \subset \mathrm{C}$, and by $\operatorname{Hol}(\Delta)$ the set of all holomorphic self-mappings of $\Delta$.

We say that a family $S=\left\{F_{t}\right\}_{t \geq 0} \subset \operatorname{Hol}(\Delta)$ is a one-parameter continuous semigroup on $\Delta$ (a semigroup, in short) if

(i) $F_{t}\left(F_{s}(z)\right)=F_{t+s}(z)$ for all $t, s \geq 0$ and $z \in \Delta$,

and

(ii) $\lim _{t \rightarrow 0^{+}} F_{t}(z)=z$ for all $z \in \Delta$.

If all the elements $F_{t}, t \geq 0$, of a semigroup $S$ are automorphisms of $\Delta$, then $S$ can be extended to a group of automorphisms $\left\{F_{t}\right\}_{t \in \mathrm{R}}$ and property (i) holds for all real $s$ and $t$.

It follows from a result of E. Berkson and H. Porta [4] that each semigroup is differentiable with respect to $t \in \mathbf{R}^{+}=[0, \infty)$. So, for each one-parameter continuous semigroup $S=\left\{F_{t}\right\}_{t \geq 0} \subset \operatorname{Hol}(\Delta)$, the limit

$$
\lim _{t \rightarrow 0^{+}} \frac{z-F_{t}(z)}{t}=f(z), \quad z \in \Delta,
$$

Received October 1, 2006, in revised form August 14, 2007. 
exists and defines a holomorphic mapping $f \in \operatorname{Hol}(\Delta, \mathrm{C})$. This mapping $f$ is called the (infinitesimal) generator of $S=\left\{F_{t}\right\}_{t \geq 0}$. Moreover, the function $u(t, z):=F_{t}(z),(t, z) \in \mathrm{R}^{+} \times \Delta$, is the unique solution of the Cauchy problem

$$
\left\{\begin{array}{l}
\frac{\partial u(t, z)}{\partial t}+f(u(t, z))=0, \\
u(0, z)=z, \quad z \in \Delta .
\end{array}\right.
$$

This solution is univalent on $\Delta$ (see [1]).

We say that $\tau \in \bar{\Delta}$ is a fixed point of $F \in \operatorname{Hol}(\Delta)$ if either $F(\tau)=\tau$, where $\tau \in \Delta$, or $\lim _{r \rightarrow 1^{-}} F(r \tau)=\tau$, where $\tau \in \partial \Delta=\{z:|z|=1\}$. If $F$ is not an automorphism of $\Delta$ with an interior fixed point, then by the Schwarz-Pick Lemma and the Julia-Wolff-Carathéodory Theorem, there is a unique fixed point $\tau \in \bar{\Delta}$ such that for each $z \in \Delta, \lim _{n \rightarrow \infty} F_{n}(z)=\tau$, where the $n$-th iteration $F_{n}$ of $F$ is defined by $F_{1}=F, F_{n}=F \circ F_{n-1}, n=2,3, \ldots$. Moreover, if $\tau \in \Delta$, then $\left|F^{\prime}(\tau)\right|<1$, and if $\tau \in \partial \Delta$, then the so-called angular derivative at the point $\tau$ (see the definition below) $F^{\prime}(\tau) \in(0,1]$. This point is called the Denjoy-Wolff point of $F$. The mapping $F$ is of

- dilation type, if $\tau \in \Delta$,

- hyperbolic type, if $\tau \in \partial \Delta$ and $0<F^{\prime}(\tau)<1$,

- parabolic type, if $\tau \in \partial \Delta$ and $F^{\prime}(\tau)=1$.

The mappings of parabolic type fall into two subclasses:

- automorphic type, if all orbits $F_{n}(z)$ are separated in the hyperbolic Poincaré metric $\rho$ of $\Delta$, i.e., $\lim _{n \rightarrow \infty} \rho\left(F_{n}(z), F_{n+1}(z)\right)>0$ for all $z \in \Delta$;

- nonautomorphic type, if no orbit $F_{n}(z)$ is hyperbolically separated, i.e., $\lim _{n \rightarrow \infty} \rho\left(F_{n}(z), F_{n+1}(z)\right)=0$ for all $z \in \Delta$.

Consider a semigroup $S=\left\{F_{t}\right\}_{t \geq 0}$ generated by $f \in \operatorname{Hol}(\Delta, \mathrm{C})$. It is a wellknown fact that all elements $F_{t}(t>0)$ of $S$ are of the same type (dilation, hyperbolic or parabolic) and have the same Denjoy-Wolff point $\tau$ which is a null point (interior or boundary) of $f$. (Recall that $\tau \in \partial \Delta$ is a boundary null point of $f \in \operatorname{Hol}(\Delta, \mathrm{C})$ if $\lim _{r \rightarrow 1^{-}} f(r \tau)=0$.) If $f$ generates a semigroup of dilation type (which does not consist of automorphisms), then $\operatorname{Re} f^{\prime}(\tau)>0$. In the hyperbolic case the angular derivative $f^{\prime}(\tau)$ defined by $f^{\prime}(\tau):=\lim _{r \rightarrow 1^{-}} \frac{f(r \tau)}{(r-1) \tau}$ exists and is a positive real number; in the parabolic case $f^{\prime}(\tau)=0$ (see, for example, [23]).

We say that a function $f \in \operatorname{Hol}(\Delta, \mathrm{C})$ has an angular limit $L$ at a point $\tau \in \partial \Delta$ and write $L:=\angle \lim _{z \rightarrow \tau} f(z)$, if $f(z) \rightarrow L$ as $z \rightarrow \tau$ in each Stolz 
angle with vertex at $\tau$ (see [20]). If $L$ is finite and the angular limit

$$
M:=\angle \lim _{z \rightarrow \tau} \frac{f(z)-L}{z-\tau}
$$

exists, then $M$ is said to be the angular derivative $f^{\prime}(\tau)$.

It is known (see [20], p. 79) that the existence of the first angular derivative $f^{\prime}(\tau)$ of a function $f \in \operatorname{Hol}(\Delta, \mathrm{C})$ is equivalent to each of the following conditions:

(1) there exists $\angle \lim _{z \rightarrow \tau} f^{\prime}(z)$, and then $f^{\prime}(\tau)=\angle \lim _{z \rightarrow \tau} f^{\prime}(z)$;

(2) the function $f$ admits the representation

$$
f(z)=a_{0}+a_{1}(z-\tau)+\gamma(z),
$$

where $\gamma \in \operatorname{Hol}(\Delta, \mathrm{C}), \angle \lim _{z \rightarrow \tau} \frac{\gamma(z)}{z-\tau}=0$, and then $f^{\prime}(\tau)=a_{1}$.

In Section 2 of this paper we show that higher order angular derivatives of $f$ can also be defined by either one of these ways and the definitions are equivalent (Proposition 2). Furthermore, we show that for a semigroup $\left\{F_{t}\right\}_{t \geq 0}$ generated by $f \in \operatorname{Hol}(\Delta, \mathrm{C})$, the existence of the $n$-th $(n>1)$ angular derivative $f^{(n)}(\tau)$ of $f$ at its boundary null point $\tau \in \partial \Delta$ implies that for each element $F_{t}$ of the semigroup, the $n$-th angular derivative at $\tau$ also exists, and obtain formulae connecting $F^{(n)}(\tau)$ with $f^{n}(\tau)$ for $n=2,3$ (Theorem 1).

Using these facts, we investigate in Sections 3, 4, and 5 conditions under which the commutativity of two given elements of the semigroups $S_{1}=\left\{F_{t}\right\}_{t \geq 0}$ and $S_{2}=\left\{G_{t}\right\}_{t \geq 0}$ implies that the semigroups commute for the dilation, hyperbolic and parabolic cases, respectively (Theorems 2, 4, and 5).

\section{Higher order boundary derivatives}

We begin by recalling the following known fact.

Proposition 1 ([20], p. 80). Let $h$ be holomorphic in $\Delta$. If $\operatorname{Im} h(z)$ has a finite angular limit at $\tau \in \partial \Delta$, then $(z-\tau) h^{\prime}(z)$ has the angular limit 0 at $\tau$.

Proposition 2. Let $f \in \operatorname{Hol}(\Delta, \mathrm{C})$ and let $\tau \in \partial \Delta$. Then the following assertions are equivalent for any integer $k \geq 0$ :

(i) The function $f$ admits the representation

$$
f(z)=\sum_{j=0}^{k} \frac{a_{j}}{j !}(z-\tau)^{j}+\gamma_{k}(z),
$$

where $\angle \lim _{z \rightarrow \tau} \frac{\gamma_{k}(z)}{(z-\tau)^{k}}=0$. 
(ii) The angular limit

$$
\angle \lim _{z \rightarrow \tau} f^{(k)}(z)
$$

exists finitely and coincides with $a_{k}$ in representation (2).

(iii) For each $0 \leq n \leq k$, the angular limit

$$
\angle \lim _{z \rightarrow \tau} f^{(n)}(z)
$$

exists finitely and coincides with $a_{n}$ in representation (2).

Proof. (i) $\Rightarrow$ (ii). Let (i) hold. We show by induction that for all $0 \leq n \leq k$, the angular limit $\angle \lim _{z \rightarrow \tau} \frac{\gamma_{k}^{(n)}(z)}{(z-\tau)^{k-n}}$ exists and equals zero, where $\gamma_{k}^{(0)}:=\gamma_{k}$ and $\gamma_{k}^{(n)}$ is the $n$-th derivative of $\gamma_{k}$.

For $n=0$ this relation is given. Suppose that it holds for $n=m-1, m \leq k$.

Denote

$$
h(z):=\frac{\gamma_{k}^{(m-1)}(z)}{(z-\tau)^{k-m+1}}+\frac{a_{k}}{(k-m+1) !}, \quad z \in \Delta .
$$

Then

$$
\angle \lim _{z \rightarrow \tau} h(z)=\frac{a_{k}}{(k-m+1) !}
$$

and, by Proposition 1,

$$
\angle \lim _{z \rightarrow \tau}(z-\tau) h^{\prime}(z)=0
$$

On the other hand, differentiating (2) $m-1$ times, we have

$$
h(z):=\frac{f^{(m-1)}(z)-\sum_{j=0}^{k-m} \frac{a_{m-1+j}}{j !}(z-\tau)^{j}}{(z-\tau)^{k-m+1}}, \quad z \in \Delta,
$$

and so

$$
(z-\tau) h^{\prime}(z)=\frac{f^{(m)}(z)-\sum_{j=0}^{k-m-1} \frac{a_{m+j}}{j !}(z-\tau)^{j}}{(z-\tau)^{k-m}}-(k-m+1) h(z), \quad z \in \Delta .
$$

Hence, there exists the angular limit

$$
\angle \lim _{z \rightarrow \tau} \frac{\gamma_{k}^{(m)}(z)}{(z-\tau)^{k-m}}=\angle \lim _{z \rightarrow \tau} \frac{f^{(m)}(z)-\sum_{j=0}^{k-m} \frac{a_{m+j}}{j !}(z-\tau)^{j}}{(z-\tau)^{k-m}}=0 .
$$


Consequently, $\angle \lim _{z \rightarrow \tau} \frac{\gamma_{k}^{(n)}(z)}{(z-\tau)^{k-n}}=0$ for all $0 \leq n \leq k$. In particular, $\angle \lim _{z \rightarrow \tau} \gamma_{k}^{(k)}(z)=0$. So, by (2), the angular limit $\angle \lim _{z \rightarrow \tau} f^{(k)}(z)$ exists finitely and coincides with $a_{k}$.

(ii) $\Rightarrow$ (iii). Suppose now that there exists the finite limit

$$
a_{k}:=\angle \lim _{z \rightarrow \tau} f^{(k)}(z)
$$

Consider the equality

$$
f^{(k-1)}(z)=f^{(k-1)}(0)+\int_{0}^{z} f^{(k)}(s) d s, \quad z \in \Delta .
$$

Since the angular limit (3) exists finitely, the function $f^{(k)}(z)$ is continuous on each curve $\Gamma(t), \alpha \leq t \leq \beta, \Gamma(\alpha)=0, \Gamma(\beta)=\tau$, strictly inside some Stolz angle at $\tau$. Hence, there exists the finite angular limit

$$
a_{k-1}:=\angle \lim _{z \rightarrow \tau} f^{(k-1)}(z)=f^{(k-1)}(0)+\int_{0}^{\tau} f^{(k)}(s) d s .
$$

Similarly, for each $0 \leq n \leq k$, the limit

$$
a_{n}:=\angle \lim _{z \rightarrow \tau} f^{(n)}(z)
$$

exists finitely.

(iii) $\Rightarrow$ (i). Now we show by induction that for each $0 \leq n \leq k$,

$$
f^{(k-n)}(z)=\sum_{j=0}^{n} \frac{a_{k-n+j}}{j !}(z-\tau)^{j}+\gamma_{n}(z)
$$

with $\angle \lim _{z \rightarrow \tau} \frac{\gamma_{n}(z)}{(z-\tau)^{n}}=0$.

For $n=0$ equality (5) follows immediately from (3). Suppose that it holds for $n=m-1(m \leq k)$, i.e.,

$$
f^{(k-m+1)}(z)=\sum_{j=0}^{m-1} \frac{a_{k-m+1+j}}{j !}(z-\tau)^{j}+\gamma_{m-1}(z),
$$

where $\angle \lim _{z \rightarrow \tau} \frac{\gamma_{m-1}(z)}{(z-\tau)^{m-1}}=0$.

It is clear that

$$
\frac{f^{(k-m)}(z)-a_{k-m}}{z-\tau}=\int_{0}^{1} f^{(k-m+1)}(t \tau+(1-t) z) d t .
$$


Therefore, by (4),

$$
\angle \lim _{z \rightarrow \tau} \frac{f^{(k-m)}(z)-a_{k-m}}{z-\tau}=\angle \lim _{z \rightarrow \tau} \int_{0}^{1} f^{(k-m+1)}(t \tau+(1-t) z) d t=a_{k-m+1} .
$$

On the other hand, by (6),

$$
\begin{aligned}
& \frac{f^{(k-m)}(z)-a_{k-m}}{z-\tau} \\
& =\int_{0}^{1} f^{(k-m+1)}(t \tau+(1-t) z) d t \\
& =\int_{0}^{1}\left(\sum_{j=0}^{m-1} \frac{a_{k-m+1+j}}{j !}(t \tau+(1-t) z-\tau)^{j}+\gamma_{m-1}(t \tau+(1-t) z)\right) d t \\
& =\sum_{j=0}^{m-1} \frac{a_{k-m+1+j}}{(j+1) !}(z-\tau)^{j}+\int_{0}^{1} \gamma_{m-1}(t \tau+(1-t) z) d t .
\end{aligned}
$$

Hence,

$$
f^{(k-m)}(z)=\sum_{j=0}^{m} \frac{a_{k-m+j}}{j !}(z-\tau)^{j}+\gamma_{m}(z),
$$

where $\gamma_{m}(z)=(z-\tau) \int_{0}^{1} \gamma_{m-1}(t \tau+(1-t) z) d t$.

Now we verify that $\angle \lim _{z \rightarrow \tau} \frac{\gamma_{m}(z)}{(z-\tau)^{m}}=0$. Indeed,

$$
\begin{aligned}
\angle \lim _{z \rightarrow \tau} & \frac{\gamma_{m}(z)}{(z-\tau)^{m}} \\
\quad & \angle \lim _{z \rightarrow \tau} \int_{0}^{1} \frac{\gamma_{m-1}(t \tau+(1-t) z)}{(z-\tau)^{m-1}} d t \\
& =\angle \lim _{z \rightarrow \tau} \int_{0}^{1} \frac{\gamma_{m-1}(t \tau+(1-t) z)}{(t \tau+(1-t) z-\tau)^{m-1}} \cdot \frac{(t \tau+(1-t) z-\tau)^{m-1}}{(z-\tau)^{m-1}} d t \\
& =\int_{0}^{1}\left((1-t)^{m-1} \angle \lim _{z \rightarrow \tau} \frac{\gamma_{m-1}(t \tau+(1-t) z)}{(t \tau+(1-t) z-\tau)^{m-1}}\right) d t=0,
\end{aligned}
$$

and for $n=m$ (5) is proved. By induction, (5) holds for all $0 \leq n \leq k$. This equality with $n=k$ yields representation (2).

Remark 1. Similar expansions to those which appear in Proposition 2 have already been used in [25] and [26].

Remark 2. The implication (i) $\Rightarrow$ (ii) of Proposition 2 does not hold if we replace the angular limit with the unrestricted one. This can be seen by 
studying the holomorphic self-mapping $f(w)=i+w+\exp (i w)$ of the upper half-plane $\{w \in C: \operatorname{Im} w>0\}$, as suggested by the referee. At the same time, repeating the proof, one can see that the implications (ii) $\Rightarrow$ (iii) $\Rightarrow$ (i) hold if we replace the angular limits by the unrestricted ones.

If $\left\{F_{t}\right\}_{t \geq 0}$ is a one-parameter continuous semigroup with a boundary fixed point $\tau \in \partial \Delta$ generated by $f$, then the angular derivatives $F_{t}^{\prime}(\tau)$ for all $t>$ 0 are finite if and only if the angular derivative $f^{\prime}(\tau)=: \beta$ exists finitely. Moreover, in this case $F_{t}^{\prime}(\tau)=e^{-\beta t}$ (see [16], [22], [17] and [9]).

As far as the higher order angular derivatives are concerned, even for the Denjoy-Wolff point one cannot assert that they do exist. Consider, for example, the parabolic holomorphic self-mapping $F$ of $\Delta$ defined by

$$
F(z):=\frac{2 z+(1-z) \log \left(\frac{2}{1-z}\right)}{2+(1-z) \log \left(\frac{2}{1-z}\right)}, \quad z \in \Delta,
$$

where Log is the principal branch of the logarithm (see ([11])). The DenjoyWolff point of this mapping is $\tau=1$. Consequently, there exists $\angle \lim _{z \rightarrow 1} \frac{d F(z)}{d z}$. However, the angular $\operatorname{limit} \angle \lim _{z \rightarrow 1} \frac{d^{2} F(z)}{d z^{2}}$ does not exist finitely.

In Theorem 1 below we show that the existence of the angular derivatives $f^{\prime \prime}(\tau)$ and $f^{\prime \prime \prime}(\tau)$ of the generator $f$ of a semigroup $\left\{F_{t}\right\}_{t \geq 0}$ at a boundary fixed point $\tau$ implies that for each $t>0$, the angular derivatives $F_{t}^{\prime \prime}(\tau):=$ $\angle \lim _{z \rightarrow \tau} \frac{\partial^{2} F_{t}(z)}{\partial z^{2}}$ and $F_{t}^{\prime \prime \prime}(\tau):=\angle \lim _{z \rightarrow \tau} \frac{\partial^{3} F_{t}(z)}{\partial z^{3}}$ also exist. Moreover, we give formulae which connect these derivatives. In the proof we use the following lemma (see [19], p. 303) which is also a consequence of Julia's classical lemma.

Lemma 1. Let $F \in \operatorname{Hol}(\Delta)$ and let $\tau \in \partial \Delta$ be a boundary fixed point of $F$. If $F$ is conformal at $\tau$, then nontangential convergence of $z$ to $\tau$ implies that $F(z)$ converges to $\tau$ nontangentially.

THEOREM 1. Let $S=\left\{F_{t}\right\}_{t \geq 0}$ be a one-parameter continuous semigroup generated by $f \in \operatorname{Hol}(\Delta, \mathrm{C})$ and let $\tau \in \partial \Delta$ be a boundary null point of $f$.

(i) If $f^{\prime}(\tau):=\angle \lim _{z \rightarrow \tau} f^{\prime}(z)$ exists finitely, then for each $t \geq 0, F_{t}^{\prime}(\tau):=$ $\angle \lim _{z \rightarrow \tau} F_{t}^{\prime}(z)$ also exists and

$$
F_{t}^{\prime}(\tau)=e^{-\beta t},
$$

where $\beta=f^{\prime}(\tau)$.

(ii) If $f^{\prime \prime}(\tau):=\angle \lim _{z \rightarrow \tau} f^{\prime \prime}(z)$ exists finitely, then for each $t \geq 0, F_{t}^{\prime \prime}(\tau):=$ $\angle \lim _{z \rightarrow \tau} F_{t}^{\prime \prime}(z)$ also exists and

$$
F_{t}^{\prime \prime}(\tau)= \begin{cases}-\alpha t, & \beta=0 \\ \frac{\alpha}{\beta} e^{-\beta t}\left(e^{-\beta t}-1\right), & \beta \neq 0,\end{cases}
$$


where $\beta=f^{\prime}(\tau), \alpha=f^{\prime \prime}(\tau)$.

(iii) If $f^{\prime \prime \prime}(\tau):=\angle \lim _{z \rightarrow \tau} f^{\prime \prime \prime}(z)$ exists finitely, then for each $t \geq 0$, $F_{t}^{\prime \prime \prime}(\tau):=\angle \lim _{z \rightarrow \tau} F_{t}^{\prime \prime \prime}(z)$ also exists and

(9) $F_{t}^{\prime \prime \prime}(\tau)= \begin{cases}\frac{3}{2} \alpha^{2} t^{2}-\gamma t, & \beta=0 \\ \left(\frac{3 \alpha^{2}}{2 \beta^{2}}+\frac{\gamma}{2 \beta}\right) e^{-3 \beta t}-3 \frac{\alpha^{2}}{\beta^{2}} e^{-2 \beta t}+\left(\frac{3 \alpha^{2}}{2 \beta^{2}}-\frac{\gamma}{2 \beta}\right) e^{-\beta t}, & \beta \neq 0,\end{cases}$

where $\beta=f^{\prime}(\tau), \alpha=f^{\prime \prime}(\tau), \gamma=f^{\prime \prime \prime}(\tau)$.

Proof. Since assertion (i) has been proved in [22] (see also [9] and [16]), we only present here proofs of assertions (ii) and (iii).

(ii) We have already mentioned above that semigroup elements solve the Cauchy problem (1). Differentiating the equality

$$
\frac{\partial F_{t}(z)}{\partial t}+f\left(F_{t}(z)\right)=0, \quad z \in \Delta, \quad t \geq 0,
$$

two times with respect to $z \in \Delta$, we get

$$
\frac{\partial}{\partial t}\left(\frac{\partial^{2} F_{t}(z)}{\partial z^{2}}\right)+f^{\prime \prime}\left(F_{t}(z)\right)\left(\frac{\partial F_{t}(z)}{\partial z}\right)^{2}+f^{\prime}\left(F_{t}(z)\right) \frac{\partial^{2} F_{t}(z)}{\partial z^{2}}=0
$$

for all $z \in \Delta$ and $t \geq 0$.

Define the functions $p(z, t):=f^{\prime}\left(F_{t}(z)\right), q(z, t):=-f^{\prime \prime}\left(F_{t}(z)\right)\left(\frac{\partial F_{t}(z)}{\partial z}\right)^{2}$ and $u_{2}(z, t):=\frac{\partial^{2} F_{t}(z)}{\partial z^{2}}, z \in \Delta, t \geq 0$. It is clear that $u_{2}(z, 0)=0$. Rewriting (11) in the form

$$
\frac{\partial u_{2}(z, t)}{\partial t}+p(z, t) u_{2}(z, t)=q(z, t), \quad z \in \Delta, \quad t \geq 0,
$$

we find

$$
u_{2}(z, t)=e^{-\int_{0}^{t} p(z, s) d s} \cdot \int_{0}^{t} q(z, s) e^{\int_{0}^{s} p(z, \varsigma) d \varsigma} d s .
$$

Now we fix $t$ and let $z$ tend to $\tau$ nontangentially in the right-hand side of this equality. Since $\angle \lim _{z \rightarrow \tau} f^{\prime \prime}(z):=\alpha$ exists finitely, by Proposition 2 , the angular limit $\angle \lim _{z \rightarrow \tau} f^{\prime}(z):=\beta$ also exists finitely. Consequently, $\tau$ is a boundary fixed point of $F_{t}$ for all $t \geq 0$ (see [22] and [9]). Moreover, by item (i), $\angle \lim _{z \rightarrow \tau} F_{t}^{\prime}(z)=e^{-\beta t} \neq 0$ for each $t \geq 0$. Hence, by Lemma 1 , $F_{t}(z)$ converges to $\tau$ nontangentially as $z$ tends to $\tau$ nontangentially for each $t>0$, and we can conclude that $\angle \lim _{z \rightarrow \tau} p(z, t)=\beta$ and $\angle \lim _{z \rightarrow \tau} q(z, t)=$ 
$-\alpha e^{-2 \beta t}$ for each $t>0$. Hence,

$$
\begin{aligned}
& \angle \lim _{z \rightarrow \tau}\left(e^{-\int_{0}^{t} p(z, t) d s} \cdot \int_{0}^{t} q(z, s) e^{\int_{0}^{s} p(z, \varsigma) d s} d s\right) \\
& \quad=e^{-\int_{0}^{t} \angle \lim _{z \rightarrow \tau} p(z, s) d s} \cdot \int_{0}^{t} \angle \lim _{z \rightarrow \tau} q(z, s) \cdot e^{\int_{0}^{s} \angle \lim _{z \rightarrow \tau} p(z, 5) d \varsigma} d s \\
& \quad=-\alpha e^{-\beta t} \int_{0}^{t} e^{-\beta s} d s .
\end{aligned}
$$

Therefore if $\beta=0$, then

$$
\angle \lim _{z \rightarrow \tau} \frac{\partial^{2} F_{t}(z)}{\partial z^{2}}=-\alpha t, \quad 0 \leq t<\infty .
$$

If $\beta \neq 0$, then

$$
\angle \lim _{z \rightarrow \tau} \frac{\partial^{2} F_{t}(z)}{\partial z^{2}}=\frac{\alpha}{\beta} e^{-\beta t} \cdot\left(e^{-\beta t}-1\right) .
$$

(iii) Differentiating equality (10) three times with respect to $z \in \Delta$, we get

$$
\begin{aligned}
\text { (12) } & \frac{\partial}{\partial t}\left(\frac{\partial^{3} F_{t}(z)}{\partial z^{3}}\right)+f^{\prime \prime \prime}\left(F_{t}(z)\right)\left(\frac{\partial F_{t}(z)}{\partial z}\right)^{3} \\
+ & 3 f^{\prime \prime}\left(F_{t}(z)\right) \frac{\partial F_{t}(z)}{\partial z} \cdot \frac{\partial^{2} F_{t}(z)}{\partial z^{2}}+f^{\prime}\left(F_{t}(z)\right) \frac{\partial^{3} F_{t}(z)}{\partial z^{3}}=0, \quad t \geq 0, z \in \Delta .
\end{aligned}
$$

Define the functions

$$
r(z, t):=-f^{\prime \prime \prime}\left(F_{t}(z)\right) \cdot\left(\frac{\partial F_{t}(z)}{\partial z}\right)^{3}-3 f^{\prime \prime}\left(F_{t}(z)\right) \cdot \frac{\partial F_{t}(z)}{\partial z} \cdot \frac{\partial^{2} F_{t}(z)}{\partial z^{2}}
$$

and $u_{3}(z, t):=\frac{\partial^{3} F_{t}(z)}{\partial z^{3}}, z \in \Delta, t \geq 0$. It is clear that $u_{3}(z, 0)=0$. Rewriting (12) in the form

$$
\frac{\partial u_{3}(t, z)}{\partial t}+p(z, t) u_{3}(z, t)=r(z, t), \quad t \geq 0,
$$

we find

$$
u_{3}(z, t)=e^{-\int_{0}^{t} p(z, s) d s} \cdot \int_{0}^{t} r(z, s) e^{\int_{0}^{s} p(z, \varsigma) d \varsigma} d s .
$$

Now we fix $t$ and let $z$ tend to $\tau$ nontangentially in the right-hand side of this equality. 
Once again, by the continuity of $p(\cdot, t)$ and $r(\cdot, t)$ in each closed Stolz angle with vertex at $\tau$,

$$
\begin{aligned}
\angle \lim _{z \rightarrow \tau} & \left(e^{-\int_{0}^{t} p(z, s) d s} \cdot \int_{0}^{t} q(z, s) e^{\int_{0}^{s} p(z, \varsigma) d \varsigma} d s\right) \\
& =e^{-\int_{0}^{t} L \lim _{z \rightarrow \tau} p(z, s) d s} \cdot \int_{0}^{t} L \lim _{z \rightarrow \tau} q(z, s) \cdot e^{\int_{0}^{s} L \lim _{z \rightarrow \tau} p(z, \varsigma) d \varsigma} d s \\
& =-e^{-\beta t} \cdot \int_{0}^{t}\left(\gamma e^{-3 \beta s}+3 \alpha e^{-\beta s} \cdot \angle \lim _{z \rightarrow \tau} \frac{\partial^{2} F_{s}(z)}{\partial z^{2}}\right) e^{\beta s} d s
\end{aligned}
$$

By Proposition 2, the limit $\angle \lim _{z \rightarrow \tau} \frac{\partial^{2} F_{t}(z)}{\partial z^{2}}$ exists and by item (ii) proved above, it is given by equality (8).

Hence, the limit $\angle \lim _{z \rightarrow \tau} \frac{\partial^{3} F_{t}(z)}{\partial z^{3}}$ exists and in the parabolic case $(\beta=0)$ it equals

$$
\angle \lim _{z \rightarrow \tau} \frac{\partial^{3} F_{t}(z)}{\partial z^{3}}=-\int_{0}^{t}\left(\gamma-3 \alpha^{2} s\right) d s=\frac{3 \alpha^{2} t^{2}}{2}-\gamma t .
$$

In the hyperbolic case $(\beta \neq 0)$ this limit also exists and

$$
\begin{aligned}
\angle \lim _{z \rightarrow \tau} \frac{\partial^{3} F_{t}(z)}{\partial z^{3}} & =-e^{-\beta t} \cdot \int_{0}^{t}\left(\left(\gamma+\frac{3 \alpha^{2}}{\beta}\right) e^{-2 \beta s}-\frac{3 \alpha^{2}}{\beta} e^{-\beta s}\right) d s \\
& =\left(\frac{3 \alpha^{2}}{2 \beta^{2}}+\frac{\gamma}{2 \beta}\right) e^{-3 \beta t}-3 \frac{\alpha^{2}}{\beta^{2}} e^{-2 \beta t}+\left(\frac{3 \alpha^{2}}{2 \beta^{2}}-\frac{\gamma}{2 \beta}\right) e^{-\beta t} .
\end{aligned}
$$

Corollary 1. Let $f \in \operatorname{Hol}(\Delta, \mathrm{C})$ be the generator of a parabolic semigroup $\left\{F_{t}\right\}_{t \geq 0}$ with the Denjoy-Wolff point $\tau \in \partial \Delta$. If $\angle \lim _{z \rightarrow \tau} f^{\prime \prime}(\tau)=$ $\angle \lim _{z \rightarrow \tau} f^{\prime \prime \prime}(\tau)=0$, then $F_{t}=$ I for all $t \geq 0$.

Indeed, these conditions imply that $F_{t}^{\prime}(\tau)=1, F_{t}^{\prime \prime}(\tau)=F_{t}^{\prime \prime \prime}(\tau)=0$ for all $t \geq 0$ and, by Corollary 2.5 in [26] (see also [8]), we get $F_{t}=\mathrm{I}$.

REMARK 3. As a matter of fact, repeating our proof and using Remark 2, one can show that the angular limits in Theorem 1 can be replaced by unrestricted limits. Namely:

Let $S=\left\{F_{t}\right\}_{t \geq 0}$ be the semigroup generated by $f$. Assume that for each $t>0$ the unrestricted limit $\lim _{z \rightarrow \tau, z \in \Delta} F_{t}(z)$ exists, where $\tau$ is a boundary null point of $f$. The following assertions hold:

(i) If the unrestricted limit $\beta:=\lim _{z \rightarrow \tau, z \in \Delta} f^{\prime}(z)$ exists finitely, then

$$
\lim _{\substack{z \rightarrow \tau \\ z \in \Delta}} F_{t}^{\prime}(z)=e^{-\beta t} \quad \text { for each } t \geq 0
$$


(ii) If the unrestricted limit $\alpha:=\lim _{z \rightarrow \tau, z \in \Delta} f^{\prime \prime}(z)$ exists finitely, then

$$
\lim _{\substack{z \rightarrow \tau \\ z \in \Delta}} F_{t}^{\prime \prime}(z)= \begin{cases}-\alpha t, & \beta=0 \\ \frac{\alpha}{\beta} e^{-\beta t}\left(e^{-\beta t}-1\right), & \beta \neq 0,\end{cases}
$$

for each $t \geq 0$.

(iii) If the unrestricted limit $\gamma:=\lim _{z \rightarrow \tau, z \in \Delta} f^{\prime \prime \prime}(z)$ exists finitely, then (14)

$$
\lim _{\substack{z \rightarrow \tau \\ z \in \Delta}} F_{t}^{\prime \prime \prime}(z)= \begin{cases}\frac{3}{2} \alpha^{2} t^{2}-\gamma t, & \beta=0 \\ \left(\frac{3 \alpha^{2}}{2 \beta^{2}}+\frac{\gamma}{2 \beta}\right) e^{-3 \beta t}-3 \frac{\alpha^{2}}{\beta^{2}} e^{-2 \beta t}+\left(\frac{3 \alpha^{2}}{2 \beta^{2}}-\frac{\gamma}{2 \beta}\right) e^{-\beta t}, & \beta \neq 0,\end{cases}
$$

for each $t \geq 0$.

REMARK 4. The arguments used in the proof of Theorem 1 can be used to derive analogous results for derivatives of any order $k \geq 4$.

\section{Semigroups with an interior fixed point}

In our proofs we use the two following facts established by C. C. Cowen in [12].

Proposition 3. Let $F, G_{1}, G_{2}$ be holomorphic self-mappings of $\Delta$, not automorphisms of $\Delta$, and let $G_{1}$ and $G_{2}$ commute with $F$. Suppose that $\tau \in \bar{\Delta}$ is the Denjoy-Wolff point of $F$ and that $0<\left|F^{\prime}(\tau)\right|<1$. Then $G_{1}$ and $G_{2}$ commute with each other.

Proposition 4. Let $F$ and $G$ be two nonconstant commuting holomorphic self-mappings of $\Delta$, not automorphisms of $\Delta$, and let $\tau \in \bar{\Delta}$ be their common Denjoy-Wolff point.

(i) If $F^{\prime}(\tau)=0$, then $G^{\prime}(\tau)=0$.

(ii) If $0<\left|F^{\prime}(\tau)\right|<1$, then $0<\left|G^{\prime}(\tau)\right|<1$.

(iii) If $F^{\prime}(\tau)=1$, then $G^{\prime}(\tau)=1$.

The following fact is more or less known (see, for example, [1]).

Proposition 5. Let $S=\left\{F_{t}\right\}_{t \geq 0}$ be a semigroup in $\Delta$. Assume $F_{t_{0}}$ is an automorphism of $\Delta$ for some $t_{0}>0$; then each element $F_{t}$ of $S$ is an automorphism of $\Delta$.

We now begin our investigation of commuting semigroups. Note that in all the following theorems the condition $F_{1} \circ G_{1}=G_{1} \circ F_{1}$ can be replaced by the condition $F_{p} \circ G_{q}=G_{q} \circ F_{p}$ for some $p, q>0$. 
Theorem 2. Let $S_{1}=\left\{F_{t}\right\}_{t \geq 0}$ and $S_{2}=\left\{G_{t}\right\}_{t \geq 0}$ be two continuous semigroups on $\Delta$ generated by $f$ and $g$, respectively, and assume that $F_{1} \circ G_{1}=$ $G_{1} \circ F_{1}$. Suppose that $f$ has an interior null point $\tau \in \Delta$. If $S_{1}$ and $S_{2}$ are not groups of automorphisms of $\Delta$, then they commute.

Proof. Since $\tau$ is an interior null point of the generator $f$, it is the unique interior fixed point of the semigroup $S_{1}$ (see [1]). The commutativity of $F_{1}$ and $G_{1}$ implies that $\tau$ is a fixed point of $G_{1}$ and, consequently, $\tau$ is a fixed point of $G_{t}$ for each $t>0$.

By our assumption, $S_{1}$ and $S_{2}$ are not groups of automorphisms of $\Delta$. By the Schwarz-Pick lemma and the univalence of $F_{t}$ and $G_{t}$ on $\Delta$, we have $0<\left|F_{t}^{\prime}(\tau)\right|<1$ and $0<\left|G_{t}^{\prime}(\tau)\right|<1$ for all $t>0$.

Then applying Proposition 3 and the semigroup property, we get that $G_{1} \circ$ $F_{t}=F_{t} \circ G_{1}$ for all $t \geq 0$. Similarly, $G_{s} \circ F_{t}=F_{t} \circ G_{s}$ for all $s, t \geq 0$.

Surprisingly, the case where $S_{1}$ contains elliptic automorphisms is more complicated. First we prove that a semigroup commuting with a group of elliptic automorphisms has a specific form.

Proposition 6. Let $S_{1}=\left\{F_{t}\right\}_{t \geq 0}$ be a nontrivial group of elliptic automorphisms of $\Delta$ with a common fixed point at $\tau \in \Delta$, and let $S_{2}=\left\{G_{t}\right\}_{t \geq 0}$ be a semigroup of self-mappings of $\Delta$. Then $S_{1}$ and $S_{2}$ commute if and only if $S_{2}$ is a semigroup of linear fractional transformations of the form

$$
G_{t}(z)=m_{\tau}\left(e^{-a t} \cdot m_{\tau}(z)\right)
$$

for some $a \in \mathrm{C}$, where $m_{\tau}(z)=\frac{\tau-z}{1-\bar{\tau} z}$.

Note that the function $G_{t}$ defined by equality (15) is a self-mapping of $\Delta$ if and only if $\operatorname{Re} a \geq 0$.

Proof. Let $S_{2}$ be of the form (15). Since both $S_{1}$ and $S_{2}$ are actually linear semigroups up to conjugation with $m_{\tau}$, they must commute.

Conversely, suppose that $F_{t} \circ G_{s}=G_{s} \circ F_{t}$ for all $s, t \geq 0$. Denote

$$
\widetilde{F}_{t}(z)=e^{i \varphi t} z, \quad \widetilde{G}_{t}=m_{\tau} \circ G_{t} \circ m_{\tau} .
$$

Then $\left\{\widetilde{F}_{t}\right\}_{t \geq 0}$ is a group of automorphisms of $\Delta$ with a fixed point at zero, and $\left\{\widetilde{G}_{t}\right\}_{t \geq 0}$ is a semigroup of self-mappings of $\Delta$ with a fixed point at zero. It is obvious that the semigroups $\left\{\widetilde{F}_{t}\right\}_{t \geq 0}$ and $\left\{\widetilde{G}_{t}\right\}_{t \geq 0}$ commute. Consequently, their generators $\widetilde{g}(z)$ and $\widetilde{f}(z)=-i \varphi z$ are proportional (see [15]). So $\widetilde{g}(z)=a z$ for some $a \in \mathrm{C}$. Therefore $\widetilde{G}_{t}(z)=e^{-a t} z$ and $G_{t}(z)=m_{\tau}\left(e^{-a t} m_{\tau}(z)\right)$.

We will see below that if $S_{1}$ is a group of elliptic automorphisms the commutativity of $F_{1}$ and $G_{1}$ does not imply that the semigroups $S_{1}$ and $S_{2}$ commute. 
Nevertheless, in this case one can still obtain some additional information about the semigroup $S_{2}$. The following assertions explain our claim.

THEOREM 3. Let $F$ be an elliptic automorphim of $\Delta$ and let $S_{2}=\left\{G_{t}\right\}_{t \geq 0}$ be a semigroup of self-mappings of $\Delta$ which are not automorphisms. Then the commutativity of $F$ and $G_{1}$ implies that $F \circ G_{t}=G_{t} \circ F$ for all $t \geq 0$.

Proof. Let $\tau \in \Delta$ be the common fixed point of $F$ and $G_{t}, t \geq 0$. Then the function $F$ is of the form $F(z)=m_{\tau}\left(e^{i \varphi} m_{\tau}(z)\right), \varphi \in \mathrm{R}, z \in \Delta$, where $m_{\tau}(z)=\frac{\tau-z}{1-\bar{\tau} z}$.

Denote $\widetilde{F}(z):=e^{i \varphi} z$ and $\widetilde{G}_{t}(z)=m_{\tau}\left(G_{t}\left(m_{\tau}(z)\right)\right)$. Then $\left\{\widetilde{G}_{t}\right\}_{t \geq 0}$ is a semigroup of self-mappings of $\Delta$ which are not automorphisms with its common fixed point at zero.

It is obvious that for each $t>0, F$ and $G_{t}$ commute if and only if $\widetilde{F}$ and $\widetilde{G}_{t}$ commute. Hence, by our assumption, $\widetilde{F} \circ \widetilde{G}_{1}=\widetilde{G}_{1} \circ \widetilde{F}$ or, which is one and the same, $e^{i \varphi} \widetilde{G}_{1}(z)=\widetilde{G}_{1}\left(e^{i \varphi} z\right)$. It follows that for all $n \in \mathrm{N}, \widetilde{F} \circ \widetilde{G}_{n}=\widetilde{G}_{n} \circ \widetilde{F}$, where $\widetilde{G}_{n}$ are the iterates of $\widetilde{G}_{1}$, i.e., $\widetilde{G}_{n}=\widetilde{G}_{1} \circ \widetilde{G}_{n-1}$.

Since $\widetilde{G}_{1}$ is a self-mapping of $\Delta$ (which is not an automorphism) with a fixed point at the origin, there exists a unique univalent solution $h$ of the functional equation

$$
h\left(\widetilde{G}_{1}(z)\right)=\alpha h(z), \quad \text { with } \quad \alpha=\widetilde{G}_{1}^{\prime}(0),
$$

normalized by $h(0)=0, h^{\prime}(0)=1$ (see, for example, [21]). This solution is given by

$$
h(z)=\lim _{n \rightarrow \infty} \frac{\widetilde{G}_{n}(z)}{\alpha^{n}} .
$$

Moreover, for all real positive $t$ (see, for instance, [14]),

$$
h\left(\widetilde{G}_{t}(z)\right)=\alpha^{t} h(z) .
$$

Therefore,

$$
\begin{aligned}
h\left(\widetilde{F}\left(\widetilde{G}_{t}(z)\right)\right) & =h\left(e^{i \varphi} \widetilde{G}_{t}(z)\right)=\lim _{n \rightarrow \infty} \frac{\widetilde{G}_{n}\left(e^{i \varphi} \widetilde{G}_{t}(z)\right)}{\alpha^{n}}=\lim _{n \rightarrow \infty} \frac{e^{i \varphi} \widetilde{G}_{n}\left(\widetilde{G}_{t}(z)\right)}{\alpha^{n}} \\
& =e^{i \varphi} h\left(\widetilde{G}_{t}(z)\right)=e^{i \varphi} \alpha^{t} h(z)=\alpha^{t} \lim _{n \rightarrow \infty} \frac{e^{i \varphi} \widetilde{G}_{n}(z)}{\alpha^{n}} \\
& =\alpha^{t} \lim _{n \rightarrow \infty} \frac{\widetilde{G}_{n}\left(e^{i \varphi} z\right)}{\alpha^{n}}=\alpha^{t} h\left(e^{i \varphi} z\right)=h\left(\widetilde{G}_{t}\left(e^{i \varphi} z\right)\right) \\
& =h\left(\widetilde{G}_{t}(\widetilde{F}(z))\right)
\end{aligned}
$$

and, by the univalence of $h$, we get $\widetilde{F} \circ \widetilde{G}_{t}=\widetilde{G}_{t} \circ \widetilde{F}$ for all $t \geq 0$. Consequently, $F$ and $G_{t}$ commute for all $t \geq 0$ as asserted. 
Corollary 2. Let $S_{1}=\left\{F_{t}\right\}_{t \geq 0}$ be a group of elliptic automorphisms of $\Delta$, i.e., $F_{t}(z)=m_{\tau}\left(e^{i \varphi t} m_{\tau}(z)\right), \varphi \in \mathrm{R}, \tau \in \Delta$, and let $S_{2}=\left\{G_{t}\right\}_{t \geq 0}$ be a semigroup of self-mappings of $\Delta$ with $G_{1} \neq I$. Suppose that $\frac{\varphi}{\pi}$ is an irrational number and $F_{1}$ and $G_{1}$ commute. Then $G_{t}(z)=m_{\tau}\left(e^{-a t} m_{\tau}(z)\right), a \in \mathrm{C}$, and, consequently, the semigroups $S_{1}$ and $S_{2}$ commute.

Proof. Once again, we define the functions $\widetilde{F}_{t}=e^{i \varphi t} z$ and $\widetilde{G}_{t}=m_{\tau} \circ$ $G_{t} \circ m_{\tau}$. The commutativity of $F_{1}$ and $G_{1}$ implies that $\widetilde{F}_{1} \circ \widetilde{G}_{1}=\widetilde{G}_{1} \circ \widetilde{F}_{1}$ and, by Proposition 6 and Theorem $3, \widetilde{F}_{1} \circ \widetilde{G}_{t}=\widetilde{G}_{t} \circ \widetilde{F}_{1}$ for all $t \geq 0$. Therefore $\widetilde{G}_{t}\left(e^{i n \varphi} z\right)=e^{i n \varphi} \widetilde{G}_{t}(z)$ for all $n \in \mathrm{N}$. Since the set $\left\{e^{i n \varphi}\right\}_{n \in \mathrm{N}}$ is dense in the unit circle, $\widetilde{G}_{t}(\lambda z)=\lambda \widetilde{G}_{t}(z)$ for all $\lambda$ with $|\lambda|=1$ and $z \in \Delta$, by the continuity of $\widetilde{G}_{t}$ on $\Delta$.

Fix $0 \neq z \in \Delta$ and $t>0$, and consider the analytic function $q(\lambda)$ on the closed unit disk defined by

$$
q(\lambda)= \begin{cases}\frac{\widetilde{G}_{t}(\lambda z)}{\lambda}, & \lambda \neq 0, \\ \lim _{\lambda \rightarrow 0} \frac{\widetilde{G}_{t}(\lambda z)}{\lambda}=\left.z \frac{\partial}{\partial w} \widetilde{G}_{t}(w)\right|_{w=0}, & \lambda=0 .\end{cases}
$$

This function is constant on the unit circle: $q(\lambda)=\widetilde{G}_{t}(z)$. Moreover, $q(\lambda) \neq 0$ for all $\lambda \in \Delta$. Therefore, $q(\lambda)=\widetilde{G}_{t}(z)$ for all $\lambda \in \bar{\Delta}$. So for each $z \neq 0$ and $t>0, \widetilde{G}_{t}(\lambda z)=\lambda \widetilde{G}_{t}(z)$. Consequently, this equality holds for all $z \in \Delta$. Hence $\widetilde{G}_{t}$ is a linear function for each $t>0$, i.e., $\widetilde{G}_{t}(z)=e^{-a t} z$ for some $a \in \mathrm{C}, \operatorname{Re} a \geq 0$, and the assertion follows.

In contrast with this corollary, if $\frac{\varphi}{\pi}$ is a rational number, the semigroups $S_{1}$ and $S_{2}$ do not necessarily commute. The following example gives a large class of semigroups $S_{2}=\left\{G_{t}\right\}_{t \geq 0}$ such that $F_{1} \circ G_{t}=G_{t} \circ F_{1}$ for all $t \geq 0$, but the semigroups $S_{1}$ and $S_{2}$ do not commute.

ExAmple. Let $S_{1}=\left\{F_{t}\right\}_{t \geq 0}$, where $F_{t}(z)=e^{i \frac{2 \pi}{n} t} z, n \in \mathrm{N}$, and let $S_{2}=$ $\left\{G_{t}\right\}_{t \geq 0}$ be the semigroup generated by $g(z)=z p\left(z^{n}\right)$, where Re $p(z) \geq 0$ for all $z \in \Delta$. Then $F_{1} \circ G_{t}=G_{t} \circ F_{1}$ for all $t \geq 0$.

Indeed, denote $u=u(t, z):=G_{t}(z)$. Then $u$ is the unique solution of the Cauchy problem

$$
\left\{\begin{array}{l}
\frac{\partial u}{\partial t}+u p\left(u^{n}\right)=0, \\
u(0, z)=z, \quad z \in \Delta,
\end{array}\right.
$$

and, consequently,

$$
\int_{z}^{G_{t}(z)} \frac{d \varsigma}{\varsigma p\left(\varsigma^{n}\right)}=-t \quad \text { for all } \quad z \in \Delta .
$$


Substituting $e^{i \frac{2 \pi}{n}} z$ instead of $z$, we get

$$
\int_{e^{i \frac{2 \pi}{n}} z}^{G_{t}\left(e^{i \frac{2 \pi}{n}} z\right)} \frac{d \varsigma}{\varsigma p\left(\varsigma^{n}\right)}=-t
$$

Now substitute $\varsigma=e^{i \frac{2 \pi}{n}} w$ :

(19)

$$
\int_{z}^{G_{t}\left(e^{i \frac{2 \pi}{n}} z\right) e^{-i \frac{2 \pi}{n}}} \frac{d w}{w p\left(w^{n} e^{i 2 \pi}\right)}=\int_{z}^{G_{t}\left(e^{i \frac{2 \pi}{n}} z\right) e^{-i \frac{2 \pi}{n}}} \frac{d w}{w p\left(w^{n}\right)}=-t, \quad z \in \Delta .
$$

Equalities (18) and (19) imply that

$$
\int_{G_{t}(z)}^{G_{t}\left(e^{i \frac{2 \pi}{n}} z\right) e^{-i \frac{2 \pi}{n}}} \frac{d w}{w p\left(w^{n}\right)}=0, \quad z \in \Delta .
$$

By the uniqueness of the solution to the Cauchy problem (17), the equation

$$
\int_{z}^{u} \frac{d w}{w p\left(w^{n}\right)}=-s, \quad s \geq 0, \quad z \in \Delta
$$

has the unique solution $u=G_{s}(z)$ for each $s \geq 0$. Thus, it follows from (20) that $G_{t}\left(e^{i \frac{2 \pi}{n}} z\right) e^{-i \frac{2 \pi}{n}}=G_{0}\left(G_{t}(z)\right)=G_{t}(z)$. Hence, $G_{t}\left(e^{i \frac{2 \pi}{n}} z\right)=e^{i \frac{2 \pi}{n}} G_{t}(z)$. Therefore, $F_{1}$ commutes with $G_{t}$ for all $t \geq 0$. At the same time, if $p$ is not a constant function, the semigroups do not commute because their generators are not proportional (see Theorem 4 in [15]).

REMARK 5. The following complement to this example has been inspired by the referee. If at least one element of a semigroup $S_{2}=\left\{G_{t}\right\}_{t \geq 0}$, say $G_{1}$, has the form $G_{1}(z)=z \phi_{1}\left(z^{n}\right), \phi_{1} \in \operatorname{Hol}(\Delta)$, then all the elements have the same form: $G_{t}(z)=z \phi_{t}\left(z^{n}\right)$ for some functions $\phi_{t} \in \operatorname{Hol}(\Delta)$; so the semigroup generator can be represented as $g(z)=z p\left(z^{n}\right)$ with $\operatorname{Re} p(z) \geq 0$.

Indeed, the representation $G_{1}(z)=z \phi_{1}\left(z^{n}\right)$ is equivalent to the commutativity of $G_{1}$ with $F_{1}(z)=e^{i \frac{2 \pi}{n}} z$. By Theorem 3 , each mapping $G_{t}, t \geq 0$, must commute with $F_{1}$. Hence $G_{t}(z)=z \phi_{t}\left(z^{n}\right), \phi_{t} \in \operatorname{Hol}(\Delta)$. Differentiating $G_{t}$ at $t=0^{+}$, we arrive at our claim.

\section{Semigroups of hyperbolic type}

We start this section with a result of M. H. Heins [18].

LeMma 2. Let $F$ be a hyperbolic automorphism of $\Delta$, and let $G \in \operatorname{Hol}(\Delta)$ $(G \neq I)$ commute with $F$. Then $G$ is also a hyperbolic automorphism of $\Delta$. 
This result can be complemented by the following assertion which is of independent interest.

Proposition 7. Let $F$ and $G$ be two commuting holomorphic self-mappings of $\Delta$ and assume that $G$ is not the identity. If $F$ is of hyperbolic type, then $G$ is of hyperbolic type too.

Proof. If $F$ is a hyperbolic automorphism of $\Delta$, then by Lemma $2, G$ is a hyperbolic automorphism of $\Delta$.

Let $F$ be a holomorphic self-mapping of $\Delta$ which is not an automorphism of $\Delta$. In this case, by a result in [2], the mappings $F$ and $G$ have a common Denjoy-Wolff point $\tau \in \partial \Delta$. We have to show that $G$ is of hyperbolic type, i.e., $0<G^{\prime}(\tau)<1$. Suppose, to the contrary, that $G$ is of parabolic type, i.e., $G^{\prime}(\tau)=1$. Then, by Proposition 4(ii), $G$ must be a parabolic automorphism.

Denote $\varphi:=C \circ F \circ C^{-1}$ and $\psi:=C \circ G \circ C^{-1}$, where $C(z)=\frac{\tau+z}{\tau-z}$. Then $f$ and $g$ are two commuting holomorphic self-mappings of the right half-plane $\mathrm{H}=\{z \in \mathrm{C}: \operatorname{Re} z>0\}$ with their common Denjoy-Wolff point at infinity. Moreover, $\psi$ is a parabolic automorphism of $\mathrm{H}$ while $\varphi$ is a hyperbolic self-mapping of $\mathrm{H}$. Consequently, $\varphi$ and $\psi$ are of the forms (see [21]):

$$
\varphi(w)=c w+\Gamma_{F}(w) \quad \text { with } \quad c=\frac{1}{F^{\prime}(\tau)}>1 \quad \text { and } \quad \angle \lim _{w \rightarrow \infty} \frac{\Gamma_{F}(w)}{w}=0,
$$

and

$$
\psi(w)=w+i b \quad \text { with } \quad b \in \mathbf{R} \backslash\{0\} \quad \text { and } \quad w \in \mathrm{H} .
$$

By a simple calculation and the commutativity of $\varphi$ and $\psi_{n}$, we infer from the above representations that

$$
\varphi(w+n i b)=\varphi(w)+n i b, \quad w \in \mathrm{H} .
$$

Hence,

$$
\frac{\varphi(w+n i b)}{w+n i b}=\frac{\varphi(w)}{w+n i b}+\frac{n i b}{w+n i b}, \quad w \in \mathrm{H} .
$$

Letting $n \rightarrow \infty$, we obtain that for each $w \in \mathrm{H}$, the $\operatorname{limit}_{n \rightarrow \infty} \frac{\varphi(w+n i b)}{w+n i b}$ exists and equals 1 .

Fix $w_{0} \in \mathrm{H}$. Consider the curve $\ell:=\left\{w_{0}+i t: t \in \mathrm{R}, \operatorname{sgn} t=\operatorname{sgn} b\right\}$. We intend to show that the $\operatorname{limit}_{\ell \ni z \rightarrow \infty} \frac{\varphi(z)}{z}$ exists and equals 1 .

To this end, fix an arbitrary $\varepsilon>0$ and take $N \in \mathrm{N}$ such that

$$
N>\frac{1}{|b|}\left(\frac{|\varphi(w)-w|}{\varepsilon}+|w|\right) \quad \text { and } \quad N>\frac{|w|}{|b|}
$$

for all $w \in\left[w_{0}, w_{0}+i b\right]$. 
Then $\left|\frac{\varphi(z)}{z}-1\right|<\varepsilon$ for all $z \in \ell$ with $\operatorname{sgn} b \cdot \operatorname{Im} z>\operatorname{sgn} b\left(\operatorname{Im} w_{0}+N b\right)$.

Indeed, if $\operatorname{sgn} b \cdot \operatorname{Im} z>\operatorname{sgn} b\left(\operatorname{Im} w_{0}+N b\right)$, then $z=\alpha+i k b$ for some $\alpha \in\left[w_{0}, w_{0}+i b\right]$ and $k \geq N$.

Hence, $k|b| \geq|\alpha|$ and $k>\frac{1}{|b|}\left(\frac{|\varphi(\alpha)-\alpha|}{\varepsilon}+|\alpha|\right)$. Consequently, $|\alpha+i k b|>$ $k|b|-|\alpha|>\frac{|\varphi(\alpha)-\alpha|}{\varepsilon}$.

Now using (21), we obtain that

$$
\left|\frac{\varphi(z)}{z}-1\right|=\left|\frac{\varphi(\alpha+k i b)}{\alpha+k i b}-1\right|=\left|\frac{\varphi(\alpha)-\alpha}{\alpha+i k b}\right|<\varepsilon .
$$

Thus $\lim _{\ell \ni z \rightarrow \infty} \frac{\varphi(z)}{z}=1$. It now follows from Lindelöf's theorem (see, for example, [23]) that $\angle \lim _{z \rightarrow \infty} \frac{\varphi(z)}{z}=1$, which contradicts our assumption. Therefore the mapping $G$ is indeed of hyperbolic type.

THEOREM 4. Let $S_{1}=\left\{F_{t}\right\}_{t \geq 0}$ and $S_{2}=\left\{G_{t}\right\}_{t \geq 0}$ be continuous semigroups on $\Delta$ generated by $f$ and $g$, respectively, and assume that $G \neq I$ and $F_{1} \circ$ $G_{1}=G_{1} \circ F_{1}$. Suppose that $f$ has a boundary null point $\tau \in \partial \Delta$, such that $f^{\prime}(\tau):=\angle \lim _{z \rightarrow \tau} f^{\prime}(z)>0$, i.e., the semigroup $S_{1}$ is of hyperbolic type. Then the semigroups $S_{1}$ and $S_{2}$ commute.

Proof. By our assumption, $\tau$ is the Denjoy-Wolff point of the semigroup $S_{1}$.

First we suppose that $S_{1}$ consists of automorphisms of $\Delta$. Since $f^{\prime}(\tau)>0$, $S_{1}$ consists of hyperbolic automorphisms of $\Delta$ and its generator $f$ is of the form

$$
f(z)=\frac{a_{1}}{\tau-\varsigma}(z-\tau)(z-\varsigma),
$$

where $a_{1}$ is a positive real number and $\varsigma$ is the second common fixed point of the semigroup $S_{1}$ (see [3]).

Again Lemma 2 and the commutativity of $F_{1}$ and $G_{1}$ imply that $G_{1}$ (hence, $G_{t}, t \geq 0$ ) are hyperbolic automorphisms of $\Delta$. Moreover, $S_{2}$ has the same fixed points $\tau$ and $\varsigma$; consequently, its generator $g$ is of the form

$$
g(z)=\frac{a_{2}}{\varsigma-\tau}(z-\tau)(z-\varsigma),
$$

where $a_{2}$ is a non-zero real number. Hence, $g(z)=-\frac{a_{2}}{a_{1}} f(z)$, and by Theorem 4 in [15], the semigroups commute.

Suppose now that the semigroup $S_{1}$ consists of self-mappings of $\Delta$ which are not automorphisms. By a result in [2], $\tau$ is the common Denjoy-Wolff point of $S_{1}$ and $S_{2}$. 
Then by Lemma 2 and Proposition 7, $S_{2}$ consists also of hyperbolic mappings which are not automorphisms. Now our theorem is seen to be a consequence of Proposition 3.

The referee has pointed out that another proof of Theorem 4 can be obtained by using Proposition 6 in [7].

Remark 6. Note that if $S_{1}$ and $S_{2}$ are commuting semigroups of hyperbolic type generated by $f$ and $g$, respectively, then $f(z)=k g(z)$, where $k=\frac{f^{\prime}(\tau)}{g^{\prime}(\tau)}$ is a real constant [15]. (This constant is positive whenever the semigroups are not groups.)

Therefore $S_{1}$ and $S_{2}$ coincide up to rescaling. In particular, if in Theorem 4 the derivatives $F_{1}^{\prime}(\tau)=e^{-f^{\prime}(\tau)}$ and $G_{1}^{\prime}(\tau)=e^{-g^{\prime}(\tau)}$ are equal, then $F_{t}(z)=$ $G_{t}(z)$ for all $t \geq 0$ and $z \in \Delta$.

\section{Semigroups of parabolic type}

For each $n=0,1, \ldots$, we denote by $C_{A}^{n}(\tau), \tau \in \Delta$, the class of functions $F \in \operatorname{Hol}(\Delta, \mathrm{C})$ which admit the representation

$$
F(z)=\sum_{k=0}^{n} a_{k}(z-\tau)^{k}+\gamma(z),
$$

where $\gamma \in \operatorname{Hol}(\Delta, C)$ and $\angle \lim _{z \rightarrow \tau} \frac{\gamma(z)}{(z-\tau)^{n}}=0$; and we say that $F \in C^{n}(\tau)$ when this expansion holds as $z \rightarrow \tau$ unrestrictedly.

To proceed we need the following auxiliary result.

Lemma 3. Let $F, G \in \operatorname{Hol}(\Delta)$ be two commuting univalent parabolic mappings and let $\tau=1$ be the Denjoy-Wolff point of $F$. If one of the following conditions

(i) $F, G \in C^{2}(1), F^{\prime \prime}(1) \neq 0, G^{\prime \prime}(1) \neq 0$;

(ii) $F, G \in C_{A}^{2}(1), G^{\prime \prime}(1) \neq 0, \operatorname{Re} F^{\prime \prime}(1)>0$;

(iii) $F, G \in C^{3}(1), F^{\prime \prime}(1)=G^{\prime \prime}(1)=0, F^{\prime \prime \prime}(1) \neq 0, G^{\prime \prime \prime}(1) \neq 0$

holds, then there exists a univalent function $\sigma \in \operatorname{Hol}(\Delta, \mathrm{C})$ such that

$$
\sigma \circ F=\sigma+1
$$

and

$$
\sigma \circ G=\sigma+\lambda \quad \text { with } \quad \lambda \in \mathrm{C}, \quad \lambda \neq 0 .
$$

Proof. Denote $\varphi=C \circ F \circ C^{-1}, \psi=C \circ G \circ C^{-1}, \varphi, \psi \in \operatorname{Hol}(\mathrm{H}, \mathrm{H})$, where $\mathrm{H}=\{z \in \mathrm{C}: \operatorname{Re} z>0\}$ and $C$ is the Cayley transformation given 
by $C(z)=\frac{1+z}{1-z}$. Then $\varphi$ and $\psi$ are commuting parabolic maps in $\operatorname{Hol}(\mathrm{H}, \mathrm{H})$ having $\infty$ as their common Denjoy-Wolff point.

Denote $w_{0}:=1, w_{n}^{0}:=\varphi_{n}(1), n=1,2, \ldots$,

$$
w_{n}:=\varphi_{n}(w), \quad w_{n} \in \mathbf{H},
$$

and

$$
h_{n}(w):=\frac{w_{n}-w_{n}^{0}}{w_{n+1}^{0}-w_{n}^{0}}, \quad w \in \mathrm{H} .
$$

Then $h_{n} \in \operatorname{Hol}(\mathrm{H}, \mathrm{C})$ and the sequence $\left\{h_{n}\right\}_{n=1}^{\infty}$ converges in the compact open topology to a holomorphic function $h \in \operatorname{Hol}(\mathrm{H}, \mathrm{C})$ such that $h \circ \varphi=h+1$ and the function $\sigma:=h \circ C$ solves equation (23) (see [10]). Since $F$ is univalent in $\Delta$, the solution $\sigma$ of Abel's equation (23) is also univalent in $\Delta$.

Suppose that (i) holds. Then the following expansions of $\varphi$ and $\psi$ at $\infty$ are satisfied (see [5]):

$$
\varphi(w)=w+F^{\prime \prime}(1)+\gamma_{\varphi}(w), \quad \lim _{w \rightarrow \infty} \gamma_{\varphi}(w)=0
$$

and

$$
\psi(w)=w+G^{\prime \prime}(1)+\gamma_{\psi}(w), \quad \lim _{w \rightarrow \infty} \gamma_{\psi}(w)=0
$$

Hence,

$$
\begin{aligned}
h_{n}(\psi(w)) & \\
& =\frac{\varphi_{n}(\psi(w))-w_{n}^{0}}{w_{n+1}^{0}-w_{n}^{0}}=\frac{\psi\left(\varphi_{n}(w)\right)-w_{n}^{0}}{w_{n+1}^{0}-w_{n}^{0}} \\
& =\frac{w_{n}+G^{\prime \prime}(1)+\gamma_{\psi}\left(w_{n}\right)-w_{n}^{0}}{w_{n+1}^{0}-w_{n}^{0}}=\frac{w_{n}-w_{n}^{0}}{w_{n+1}^{0}-w_{n}^{0}}+\frac{G^{\prime \prime}(1)+\gamma_{\psi}\left(w_{n}\right)}{w_{n+1}^{0}-w_{n}^{0}} \\
& =h_{n}(w)+\frac{G^{\prime \prime}(1)+\gamma_{\psi}\left(w_{n}\right)}{F^{\prime \prime}(1)+\gamma_{\varphi}\left(w_{n}\right)} \cdot \frac{F^{\prime \prime}(1)+\gamma_{\varphi}\left(w_{n}\right)}{w_{n+1}^{0}-w_{n}^{0}} .
\end{aligned}
$$

Letting $n \rightarrow \infty$, we obtain

$$
h(\psi(w))-h(w)=\frac{G^{\prime \prime}(1)}{F^{\prime \prime}(1)} \cdot \lim _{n \rightarrow \infty} \frac{F^{\prime \prime}(1)+\gamma_{\varphi}\left(w_{n}\right)}{w_{n+1}^{0}-w_{n}^{0}} .
$$

Repeating this calculation with $\varphi$ instead of $\psi$, we find that

$$
h(\varphi(w))=h(w)+\lim _{n \rightarrow \infty} \frac{F^{\prime \prime}(1)+\gamma_{\varphi}\left(w_{n}\right)}{w_{n+1}^{0}-w_{n}^{0}} .
$$


At the same time, $h \circ \varphi=h+1$. Hence $\lim _{n \rightarrow \infty} \frac{F^{\prime \prime}(1)+\gamma_{\varphi}\left(w_{n}\right)}{w_{n+1}^{0}-w_{n}^{0}}=1$.

Rewrite (27) as follows:

$$
h(\psi(w))-h(w)=\lambda, \quad \text { where } \quad \lambda=\frac{G^{\prime \prime}(1)}{F^{\prime \prime}(1)} \neq 0 \quad \text { and } \quad w \in \mathbf{H} .
$$

Substituting $h=\sigma \circ C^{-1}$ and $\psi=C \circ G \circ C^{-1}$ in the last equality we get (24).

If (ii) holds, then Theorem 14 in [11] implies that for each $z \in \Delta$, the sequence $\left\{F_{n}(z)\right\}_{n=1}^{\infty}$ converges to 1 (and, consequently, $\left\{w_{n}\right\}$ converges to $\infty$ ) nontangentially. So, in this case, one can repeat the proof of item (i), replacing the unrestricted limits in (25) and (26) by the angular limits.

Suppose now that (iii) holds. Then the following expansions of $\varphi$ and $\psi$ at $\infty$ hold (see [5]):

$$
\varphi(w)=w-\frac{2}{3} \frac{F^{\prime \prime \prime}(1)}{w+1}+\Gamma_{\varphi}(w), \quad \lim _{w \rightarrow \infty} \Gamma_{\varphi}(w) w=0
$$

and

$$
\psi(w)=w-\frac{2}{3} \frac{G^{\prime \prime \prime}(1)}{w+1}+\Gamma_{\psi}(w), \quad \lim _{w \rightarrow \infty} \Gamma_{\psi}(w) w=0 .
$$

Therefore

$$
\begin{aligned}
h_{n}(\varphi(w))=\frac{\varphi\left(w_{n}\right)-w_{n}^{0}}{w_{n+1}^{0}-w_{n}^{0}} & =\frac{w_{n}-w_{n}^{0}}{w_{n+1}^{0}-w_{n}^{0}}+\frac{-\frac{2}{3} \frac{F^{\prime \prime \prime}(1)}{w_{n}+1}+\Gamma_{\varphi}\left(w_{n}\right)}{w_{n+1}^{0}-w_{n}^{0}} \\
& =h_{n}(w)+\frac{-\frac{2}{3} \frac{F^{\prime \prime \prime}(1)}{w_{n}+1}+\Gamma_{\varphi}\left(w_{n}\right)}{w_{n+1}^{0}-w_{n}^{0}} .
\end{aligned}
$$

Letting $n \rightarrow \infty$, we obtain

$$
h(\varphi(w))=h(w)+\lim _{n \rightarrow \infty} \frac{-\frac{2}{3} \frac{F^{\prime \prime \prime}(1)}{w_{n}+1}+\Gamma_{\varphi}\left(w_{n}\right)}{w_{n+1}^{0}-w_{n}^{0}} .
$$

On the other hand, $h(\varphi(w))=h(w)+1$. Hence,

$$
\lim _{n \rightarrow \infty} \frac{-\frac{2}{3} \frac{F^{\prime \prime \prime}(1)}{w_{n}+1}+\Gamma_{\varphi}\left(w_{n}\right)}{w_{n+1}^{0}-w_{n}^{0}}=1 .
$$


Now using (29), we find

$$
\begin{aligned}
h_{n}(\psi(w)) & =\frac{\psi\left(w_{n}\right)-w_{n}^{0}}{w_{n+1}^{0}-w_{n}^{0}}=\frac{w_{n}-\frac{2}{3} \frac{G^{\prime \prime \prime}(1)}{w_{n}+1}+\Gamma_{\psi}\left(w_{n}\right)-w_{n}^{0}}{w_{n+1}^{0}-w_{n}^{0}} \\
& =h_{n}(w)+\frac{-\frac{2}{3} G^{\prime \prime \prime}(1)+\Gamma_{\psi}\left(w_{n}\right)\left(w_{n}+1\right)}{-\frac{2}{3} F^{\prime \prime \prime}(1)+\Gamma_{\varphi}\left(w_{n}\right)\left(w_{n}+1\right)} \cdot \frac{-\frac{2}{3} \frac{F^{\prime \prime \prime}(1)}{w_{n}+1}+\Gamma_{\varphi}\left(w_{n}\right)}{w_{n+1}^{0}-w_{n}^{0}} .
\end{aligned}
$$

Letting $n \rightarrow \infty$ and using (30), we get

$$
h(\psi(w))-h(w)=\lambda, \quad w \in \mathrm{H}, \quad \text { where } \quad \lambda=\frac{G^{\prime \prime \prime}(1)}{F^{\prime \prime \prime}(1)} \neq 0 .
$$

Consequently, $\sigma \circ G-\sigma=\lambda$.

Following [10], we say that the function $\sigma$ mentioned in the lemma is the Konigs intertwining function associated with $F$.

REMARK 7. The function $\sigma$ in Lemma 3 is completely determined by the function $F$. It does not depend on $G$. So if the conditions of the lemma hold for the same function $F$ and another function $G_{1} \in \operatorname{Hol}(\Delta)$, then we have the equality

$$
\sigma \circ G_{1}=\sigma+\lambda_{1}
$$

with the same function $\sigma$ and a constant $\lambda_{1} \neq 0$.

THEOREM 5. Let $S_{1}=\left\{F_{t}\right\}_{t \geq 0}$ and $S_{2}=\left\{G_{t}\right\}_{t \geq 0}$ be two non-trivial continuous semigroups on $\Delta$ generated by $f$ and $g$, respectively, and let $F_{1} \circ G_{1}=G_{1} \circ F_{1}$.

Suppose that $\tau=1$ is the boundary null point of $f$ such that $f^{\prime}(1)=0$. If either one of the following conditions

(i) the semigroups $S_{1}$ and $S_{2}$ are of non-automorphic type;

(ii) $S_{1}, S_{2} \subset C^{0}(1)$, the unrestricted limits $\alpha:=\lim _{z \rightarrow 1} f^{\prime \prime}(z)$ and $\widetilde{\alpha}:=$ $\lim _{z \rightarrow 1} g^{\prime \prime}(z)$ exist and are different from zero;

holds, then the semigroups commute.

Proof. Since $\tau=1$ is a boundary null point of $f$ and $f^{\prime}(1)=0$, it is the common Denjoy-Wolff point of the semigroup $S_{1}$. The commutativity of $F_{1}$ and $G_{1}$ implies that $\tau=1$ is the Denjoy-Wolff point of $G_{1}$ (see [2]) and, consequently, it is also the common Denjoy-Wolff point of the semigroup $S_{2}$.

Suppose that condition (i) holds. Consider the holomorphic function $\sigma$ defined by

$$
\sigma^{\prime}(z)=\frac{-1}{f(z)}, \quad \sigma(0)=0 .
$$


It follows by the Berkson-Porta formula for generators (see [4]) that $f$ can be presented in the form $f(z)=-(1-z)^{2} p(z)$, where $\operatorname{Re} p(z) \geq 0$. Thus

$$
\operatorname{Re} \frac{\sigma^{\prime}(z)}{q^{\prime}(z)} \geq 0,
$$

where $q(z)=\frac{z}{1-z}$ is a univalent convex function. Then the function $\sigma$ is close-to-convex, hence univalent in the open unit disk $\Delta$ (see, for example, Theorem 2.17 in [13]).

In addition, it can be shown (see, for example, [24] and [14]) by using the Cauchy problem (1) and (31) that the function $\sigma$ satisfies the following functional equation:

$$
\sigma\left(F_{t}(z)\right)=\sigma(z)+t
$$

Define now a univalent function $\tilde{\sigma}$ in the unit disk by $\tilde{\sigma}=\sigma \circ G_{1}$. It follows from (32) with $t=1$ and the commutativity of $F_{1}$ and $G_{1}$ that

$$
\widetilde{\sigma}\left(F_{1}(z)\right)=\widetilde{\sigma}(z)+1 .
$$

Then by Theorem 3.1 in [10], there exists a constant $\lambda \in \mathrm{C}$ such that $\widetilde{\sigma}=\sigma+\lambda$, i.e.,

$$
\sigma\left(G_{1}(z)\right)=\sigma(z)+\lambda
$$

Note that if $\lambda=0$, the univalence of $\sigma$ implies that $G_{1}(z) \equiv z$. In this case all the functions $G_{t}, t \geq 0$, coincide with the identity mapping. Hence the semigroups $S_{1}$ and $S_{2}$ commute. Therefore we can suppose that $\lambda \neq 0$.

Consider now the holomorphic function $\sigma_{1}$ defined by

$$
\sigma_{1}^{\prime}(z)=\frac{-1}{g(z)}, \quad \sigma_{1}(0)=0 .
$$

As above, $\sigma_{1}$ is univalent and satisfies the functional equation

$$
\sigma_{1}\left(G_{t}(z)\right)=\sigma_{1}(z)+t
$$

In particular, $\sigma_{1}$ is the Kœnigs intertwining function associated with $G_{1}$,

$$
\sigma_{1}\left(G_{1}(z)\right)=\sigma_{1}(z)+1 .
$$

Comparing equations (33) and (35) and using again Theorem 3.1 in [10], we obtain that $\sigma=\lambda \sigma_{1}+\mu$ for some complex number $\mu$. Differentiating the last equality, we obtain by (31) and (34), that $g(z)=\lambda f(z)$. So, by a result in [15], the semigroups $S_{1}$ and $S_{2}$ commute. 
Suppose now that condition (ii) holds. By Remark 3 above, it follows that for each $t \geq 0$,

$$
\lim _{z \rightarrow 1} F_{t}^{\prime \prime}(z)=-\alpha t
$$

and

$$
\lim _{z \rightarrow 1} G_{t}^{\prime \prime}(z)=-\widetilde{\alpha} t
$$

We have already seen in the proof of Lemma 3 that

$$
\sigma\left(G_{1}(z)\right)-\sigma(z)=\frac{G_{1}^{\prime \prime}(1)}{F_{1}^{\prime \prime}(1)} .
$$

Since $\operatorname{Re} F_{1}^{\prime \prime}(1)=0$ and $\operatorname{Re} G_{1}^{\prime \prime}(1)=0$ (see Theorem 4.4 in [5]), it follows that $\frac{G_{1}^{\prime \prime}(1)}{F_{1}^{\prime \prime}(1)} \in \mathbf{R} \backslash\{0\}$. Moreover, by Remark 3,

$$
F_{t}^{\prime \prime}(1)=-\alpha t \quad \text { and } \quad G_{t}^{\prime \prime}(1)=-\widetilde{\alpha} t, \quad t>0,
$$

where $\alpha=f^{\prime \prime}(1) \neq 0$ and $\widetilde{\alpha}=g^{\prime \prime}(1) \neq 0$. So equality (36) has the form

$$
\sigma\left(G_{1}(z)\right)-\sigma(z)=p, \quad \text { where } \quad p:=\frac{\widetilde{\alpha}}{\alpha} .
$$

On the other hand,

$$
\sigma\left(F_{t}(z)\right)-\sigma(z)=\frac{F_{t}^{\prime \prime}(1)}{F_{1}^{\prime \prime}(1)}=\frac{\alpha t}{\alpha}=t \quad \text { for all } \quad t \geq 0 .
$$

First we suppose that $p>0$. From (37) and (38) we have $\sigma\left(G_{1}(z)\right)=$ $\sigma\left(F_{p}(z)\right), z \in \Delta$, and by the univalence of $\sigma$ on $\Delta, G_{1}(z)=F_{p}(z)$ for all $z \in \Delta$. Hence, $G_{1} \circ F_{t}=F_{t} \circ G_{1}$ for all $t \geq 0$.

Fix $t>0$ and repeat these considerations with $G_{1}, F_{t}, G_{s}$ and $\widetilde{\sigma}$ instead of $F_{1}, G_{1}, F_{t}$ and $\sigma$, respectively. Namely,

$$
\widetilde{\sigma}\left(F_{t}(z)\right)-\widetilde{\sigma}(z)=\frac{F_{t}^{\prime \prime}(1)}{G_{1}^{\prime \prime}(1)}=\frac{\alpha t}{\widetilde{\alpha}}>0
$$

and

$$
\tilde{\sigma}\left(G_{s}(z)\right)-\widetilde{\sigma}(z)=\frac{G_{s}^{\prime \prime}(1)}{G_{1}^{\prime \prime}(1)}=s \quad \text { for all } \quad s>0 .
$$

Denote $\widetilde{s}:=\frac{\alpha t}{\widetilde{\alpha}}>0$. Then $\widetilde{\sigma}\left(F_{t}(z)\right)=\widetilde{\sigma}\left(G_{\widetilde{s}}(z)\right), z \in \Delta$. By the univalence of $\tilde{\sigma}$ on $\Delta$ we have $F_{t}(z)=G_{\widetilde{s}}(z)$. Therefore $G_{s} \circ F_{t}=F_{t} \circ G_{s}$ for all $s>0$. Since $t>0$ is arbitrary, it follows that the semigroups $S_{1}=\left\{F_{t}\right\}_{t \geq 0}$ and $S_{2}=\left\{G_{s}\right\}_{s \geq 0}$ commute. 
Let now $p<0$. Then by (38), $\sigma\left(F_{-p}(z)\right)-\sigma(z)=-p$ for all $z \in \Delta$. Hence, by (37),

$$
\sigma\left(F_{-p}\left(G_{1}(z)\right)\right)-\sigma\left(G_{1}(z)\right)=\sigma(z)-\sigma\left(G_{1}(z)\right), \quad z \in \Delta,
$$

and, therefore,

$$
\sigma\left(F_{-p}\left(G_{1}(z)\right)\right)=\sigma(z), \quad z \in \Delta .
$$

By the univalence of $\sigma$ on $\Delta, F_{-p}\left(G_{1}(z)\right)=z$. Consequently, $F_{-p}=G_{1}^{-1}$ on $G_{1}(\Delta)$. Since $F_{-p} \in \operatorname{Hol}(\Delta), G_{1}^{-1}$ is well defined on $\Delta$ and so $G_{1}$, as well as $F_{-p}$, are automorphisms of $\Delta$. Therefore, by Proposition $5,\left\{F_{t}\right\}_{t \geq 0}$ is a semigroup of automorphisms. Consequently, it can be extended to a group $S_{F}=\left\{F_{t}\right\}_{t \in \mathrm{R}}$ and $G_{1}=F_{p}^{-1}=F_{-p} \in S_{F}$. In particular, $G_{1} \circ F_{t}=F_{t} \circ G_{1}$ for all $t \geq 0$.

Fix $t>0$. In a similar way, using the commutativity of $F_{t}$ and $G_{1}$, one can show that the semigroup $\left\{G_{s}\right\}_{s \geq 0}$ can be extended to a group $S_{G}=\left\{G_{s}\right\}_{s \in \mathrm{R}}$ and that $F_{t} \circ G_{s}=G_{s} \circ F_{t}$ for all $s, t \in \mathrm{R}$.

ACKNOWLEDGMENT. The third author was partially supported by the Fund for the Promotion of Research at the Technion and by the Technion VPR Fund - B. and G. Greenberg Research Fund (Ottawa). All the authors are very grateful to the referee for a very detailed and careful report and for many useful suggestions and comments.

\section{REFERENCES}

1. Abate, M., The infinitesimal generators of semigroups of holomorphic maps, Ann. Mat. Pura Appl. 161 (1992), 167-180.

2. Behan, D. F., Commuting analytic functions without fixed points, Proc. Amer. Math. Soc. 37 (1973), 114-120.

3. Berkson, E., Kaufman, R., and Porta, H., Möbius transformations of the disc and oneparameter groups of isometries of $H^{p}$, Trans. Amer. Math. Soc. 199 (1974), 223-239.

4. Berkson, E., and Porta, H., Semigroups of analytic functions and composition operators, Michigan Math. J. 25 (1978), 101-115.

5. Bourdon, P. S., and Shapiro, J. H., Cyclic phenomena for composition operators, Mem. Amer. Math. Soc. 125 (1997), No. 596.

6. Bracci, F., Contreras, M. D., and Díaz-Madrigal, S., Infinitesimal generators associated with semigroups of linear fractional maps, Preprint, arXiv:math.CV/0601665.

7. Bracci, F., and Poggi-Corradini, P., On Valiron's theorem, Rep. Univ. Jyväskylä Dept. Math. Stat. 92 (2003), 39-55.

8. Bracci, F., Tauraso, R., and Vlacci, F., Identity principles for commuting holomorphic selfmaps of the unit disc, J. Math. Anal. Appl. 270 (2002), 451-473.

9. Contreras, M. D., Díaz-Madrigal, S., and Pommerenke, Ch., On boundary critical points for semigroups of analytic functions, Math. Scand. 98 (2006), 125-142.

10. Contreras, M. D., Díaz-Madrigal, S., and Pommerenke, Ch., Some remarks on the Abel equation in the unit disk, Preprint, 2005. 
11. Contreras, M. D., Díaz-Madrigal, S., and Pommerenke, Ch., Second angular derivatives and parabolic iteration in the unit disk, Preprint, 2005.

12. Cowen, C. C., Commuting analytic functions, Trans. Amer. Math. Soc. 283 (1984), 685-695.

13. Duren, P., Univalent Functions, Springer-Verlag, New York, 1983.

14. Elin, M., Goryainov, V., Reich, S., and Shoikhet, D., Fractional iteration and functional equations for functions analytic in the unit disk, Comput. Methods Funct. Theory 2 (2002), 353-366.

15. Elin, M., Levenshtein, M., Shoikhet, D., and Tauraso, R., Rigidity of holomorphic generators and one-parameter semigroups, Dynam. Systems Appl. 16 (2007), 251-266.

16. Elin, M., and Shoikhet, D., Dynamic extension of the Julia-Wolff-Carathéodory theorem, Dynam. Systems Appl. 10 (2001), 421-438.

17. Elin, M., Shoikhet, D., and Volkovich, V., Semigroups of holomorphic mappings on the unit disk with a boundary fixed point, Int. J. Pure Appl. Math. 12 (2004), 427-453.

18. Heins, M. H., A generalization of the Aumann-Carathéodory "Starrheitssatz", Duke Math. J. 8 (1941), 312-316.

19. Pommerenke, Ch., Univalent Functions, Vandenhoeck and Ruprecht, Göttingen, 1975.

20. Pommerenke, Ch., Boundary Behavior of Conformal Maps, Springer, Berlin, 1992.

21. Shapiro, J. H., Composition Operators and Classical Function Theory, Springer, Berlin, 1993.

22. Shoikhet, D., Representations of holomorphic generators and distortion theorems for spirallike functions with respect to a boundary point, Int. J. Pure Appl. Math. 5 (2003), 335-361.

23. Shoikhet, D., Semigroups in Geometrical Function Theory, Kluwer, Dordrecht, 2001.

24. Siskakis, A., Semigroups of composition operators on spaces of analytic functions, a review, Contemp. Math. 213 (1998), 229-252.

25. Tauraso, R., Commuting holomorphic maps of the unit disk, Ergodic Theory Dynam. Systems 24 (2004), 945-953.

26. Tauraso, R., and Vlacci, F., Rigidity at the boundary for holomorphic self-maps of the unit disk, Complex Variables Theory Appl. 45 (2001), 151-165.

DEPARTMENT OF MATHEMATICS

ORT BRAUDE COLLEGE

P.O. BOX 78

21982 KARMIEL

ISRAEL

E-mail: mark.elin@gmail.com, davs27@netvision.net.il
DEPARTMENT OF MATHEMATICS THE TECHNION - ISRAEL INSTITUTE OF TECHNOLOGY

32000 HAIFA

ISRAEL

E-mail: marlev@list.ru, sreich@tx.technion.ac.il 